\title{
Short-Term Effects of Muscular Denervation and Fasciotomy on Global Limb Variables during Locomotion in the Decerebrate Cat
}

\author{
Victoria A. Stahl T. Richard Nichols \\ Schools of Applied Physiology and Biomedical Engineering, Georgia Institute of Technology and Emory University, \\ Atlanta, Ga., USA
}

\section{Key Words}

Crural fascia $\cdot$ Triceps surae $\cdot$ Locomotion

\begin{abstract}
The motor system is capable of preserving the trajectories during locomotion of task level variables such as limb length and limb orientation in the face of paralysis of major muscle groups. This compensation is accomplished by the adjustment of the kinematics of joints other than the one most affected by the paralysis. The conservation of these task level variables could be accomplished quickly by feedback regulation or intrinsic mechanics, or by a longer-term adaptive process. We investigated the immediate effects of denervation of the triceps surae muscles in one limb of stepping, decerebrate cats to determine whether task level variables were preserved by short-term regulatory or intrinsic mechanisms. We further investigated the effects of disruption of the crural fascia in conjunction with denervation of the triceps surae muscles to determine whether the system consisting of multi-articular muscles of the thigh and crural fascia provided some contribution toward the preservation of limb length and orientation. Denervation led to substantial increases in ankle yield during stance, as previously observed, but also to significant decreases in limb length during early stance. Disruption of the crural fascia did not lead to increased ankle yield but, instead, to evidence for decreased propulsion. The
\end{abstract}

results suggest that the preservation of task level variables observed in other studies does not result from online error correction or intrinsic properties of the musculoskeletal system but, by inference, from longer-term neural adaptation.

Copyright $\odot 2011$ S. Karger AG, Basel

\section{Introduction}

The central nervous system has a remarkable capacity for compensation following damage to the neuromuscular system. For example, denervation of the synergists of the medial gastrocnemius muscle in the cat resulted in an increase in ankle yield during early stance for level walking and in decreased ankle extension, related to propulsion, in late stance immediately following surgery and for a few days thereafter [Pearson et al., 1999]. Although sev-

\begin{tabular}{ll} 
Abbreviations used in this paper \\
\hline EMG & electromyographic \\
FHL & flexor hallucis longus muscle \\
LG & lateral gastrocnemius \\
MG & medial gastrocnemius \\
Plant & plantaris muscle
\end{tabular}


eral of the major ankle extensors were paralyzed, ankle extension began to improve within 1 day, and weight support improved over the course of 1 week. These changes were accompanied by corresponding increases in the electromyographic (EMG) activity of the spared medial gastrocnemius muscle. Furthermore, in studies concerning the injury and repair of the nerves to the lateral (LG) and medial (MG) heads of the gastrocnemius muscles, recovery of the kinematics before the reinnervation of the muscles was observed [Maas et al., 2007]. In this latter study, it was also found that hip height and limb orientation were minimally affected only 1 week after the surgical transection and repair of the muscle nerves, suggesting preservation of more global kinematic parameters before any substantial reinnervation could have taken place. Knee extension was found to increase, contributing to the compensation for increased ankle yield.

In a more recently published study [Chang et al., 2009] in chronically prepared animals, limb length and orientation were preserved during treadmill locomotion 1 month after transection and repair of the nerves to the entire triceps surae muscle group, despite pronounced increases in ankle yield during stance. In the month following surgery, the triceps surae muscles would not have been substantially reinnervated and therefore still paralyzed. One year or more after transection and repair of the triceps surae nerves and reinnervation of these muscles, ankle yield was still pronounced during downslope walking, presumably due to the long-term disruption of proprioceptive feedback [Abelew et al., 2000]. It was also observed in this latter study that knee extension was enhanced, tending to preserve the presurgical trajectory of limb length. An important question arising from consideration of these investigations is whether task level variables are preserved immediately, despite marked changes in individual joint angles, by biomechanical or spinal regulatory mechanisms, or by adaptive neural processes as suggested by Pearson et al. [1999] for changes in individual joint angle.

A structure that could provide information for the central nervous system concerning limb length is the complex consisting of the hamstring muscles and the crural fascia. This fascia invests the triceps surae muscles and also provides a mechanical link between the biarticular muscles crossing the hip and knee and the calcaneus, making these muscles effectively triarticular [van Ingen Schenau, 1994; Stahl, 2010]. A recent study has shown that the crural fascia is important for stability of the limb during locomotion and contributes to propulsion [Stahl, 2010]. Sensory feedback from the hamstring muscles working through the crural fascia, or the crural fascia itself, could provide feedback for the regulation of task level variables in the limb and help compensate for the loss of the triceps surae muscles.

The overall hypothesis of the work presented herein was that the compensatory mechanisms that preserve task level variables for the limb following paralysis of the triceps surae muscles consist of the immediate actions of neurally mediated error correction or intrinsic mechanical properties of the musculoskeletal system. A secondary hypothesis was that sensory feedback from the retractor muscles of the thigh and crural fascia can provide the central nervous system with global information concerning limb length, and that this information could be used to accomplish on-line error correction of the limb length during locomotion. In order to test these hypotheses, we undertook an investigation with the following three objectives. The first objective was to provide a baseline measurement of the immediate or acute effects of denervation of the triceps surae muscles during locomotion against which any adaptations could be evaluated. The second objective was to test whether these immediate effects included preservation of the task level variables of limb length and orientation, and whether any such compensation was related to EMG activity in synergistic muscles. The third objective was to compare the effects of crural fasciotomy and triceps denervation on the task level variables and test whether the crural fascia can mitigate the effects of triceps denervation on coordination by virtue of the linkages that this structure provides between the hamstring muscles and the ankle. We addressed these objectives using the decerebrate cat preparation during spontaneous treadmill locomotion, as this preparation allows for the immediate evaluation of any deficits following the treatment, and therefore, avoiding any adaptive processes that might have occurred during surgical recovery or from the effects of pain. We also measured EMG activity of synergistic muscles during these procedures to test for additional evidence of short-term compensation. We found that denervation resulted in an immediate reduction in limb length in addition to pronounced ankle yield and in no change in the activation of synergistic muscles. Subsequent fasciotomy had little effect on ankle joint yield and limb length, but did result in a reduction in ankle joint acceleration during paw off. These results led us to reject our hypotheses and argue that compensation for muscle paralysis is more likely to result from longer-term adaptive changes in the central nervous system. These results have appeared in abstract form [Stahl and Nichols, 2008] and in a doctoral thesis [Stahl, 2010]. 
Fig. 1. Locations of the markers are shown in the anatomical drawing of the cat on the right. The axes of the Vicon system are shown at the bottom, where yz is the frontal plane and $\mathrm{xz}$ is the sagittal plane. The included angles and limb angle and length are defined at the top left. Limb length is denoted by the dashed line.

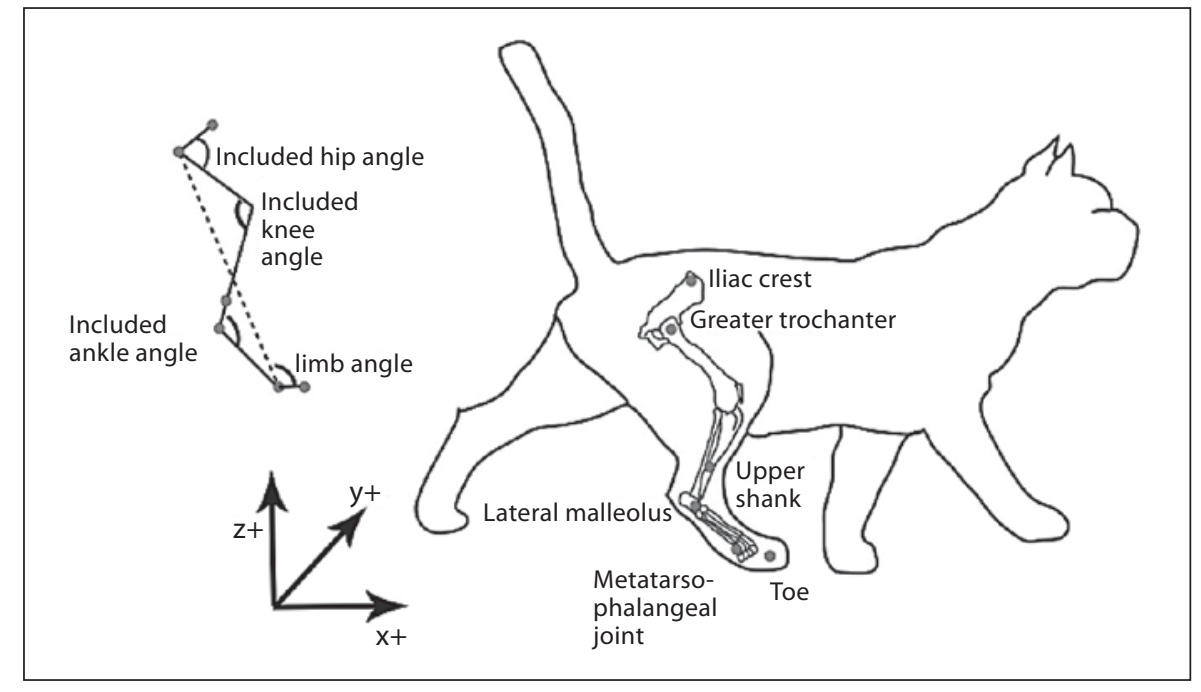

\section{Methods}

\section{Surgical Procedures}

The roles of the triceps surae and the crural fascia were investigated in 6 decerebrate cats walking slowly at approximately 0.7 $\mathrm{m} / \mathrm{s}$ atop a variable speed treadmill. All experimental protocols were approved by the Emory University and Georgia Institute of Technology Institutional Animal Care and Use Committee.

For each experiment, the animal was initially anesthetized using isoflurane gas. While under anesthesia, the skin over the gastrocnemius muscle in the right hindlimb was longitudinally split from the popliteal fat pad behind the knee to within $1 \mathrm{~cm}$ of the calcaneus. The skin was very carefully blunt dissected off the crural fascia and resealed using a flat-edged alligator clip. The animal was then supported atop a variable speed treadmill in a natural stance by affixing the head to a stereotaxic frame and clamping the base of the tail. A premammillary decerebration was performed where the brainstem was transected at a $45^{\circ}$ angle beginning rostral to the superior colliculi and ending rostral to the mammillary bodies allowing for the preservation of the subthalamic nucleus. All brain matter rostral and lateral to the transection was removed. The anesthetic was then slowly titrated until eliminated. When the animal exhibited spontaneous stepping, the treadmill was turned on and consistent stepping was observed. Occasionally, manual stimulation at the base of the tail or locomotor-like movement of the forelimbs was used to encourage a consistent stepping pattern. Once consistent stepping was elicited, all data collection was generally completed within $1 \mathrm{~h}$ of onset. At the end of the experiment, a lethal dose of concentrated pentobarbital was administered to the animal and a pneumothorax performed.

\section{Kinematic Data and Experimental Protocol}

Positions of the segment endpoint markers were recorded from the spontaneously stepping, premammillary cat using $6 \mathrm{Vi}$ con cameras collecting at $125 \mathrm{~Hz}$ and used to calculate 3D kinematics. While the cat was still under anesthesia, kinematic markers were placed on the right limb at the iliac crest, greater trochanter, upper shank (to calculate the virtual knee), lateral malleolus, metatarsophalangeal joint and toe (fig. 1). The virtual knee was calculated post-hoc in Matlab as described in Abelew et al. [2000]. The joint included angles for the hip, knee and ankle were calculated as shown in figure 1. The limb angle was calculated as the angle from the metatarsophalangeal joint to the greater trochanter in the frontal and sagittal planes. The limb length was determined as the length from the greater trochanter to the metatarsophalangeal joint. Paw contact and paw off were demarcated posthoc by video analysis.

The treadmill speed was adjusted to correspond to a slow walking velocity of $0.7 \mathrm{~m} / \mathrm{s}$. Denervation of the triceps surae muscles and crural fasciotomy were performed sequentially after control measurements were made. In one set of experiments, the muscles were denervated first. In the other set, fasciotomy was performed first. Denervation of the triceps surae muscles was accomplished by transection of the MG and LG and soleus nerves. Complete fasciotomy consisted of splitting the crural fascia longitudinally followed by transection of the medial and lateral tendinous bands. After each disruption, the skin was resealed with the alligator clip. A minimum of 3 trials was recorded under each aforementioned condition, whereby a trial is defined as a consistent, spontaneous period of walking for at least $20 \mathrm{~s}$.

\section{Kinematic Analysis}

The kinematic marker data were analyzed using custom Matlab scripts and each marker was low pass filtered with a 4th-order, zero-lag Butterworth filter at $6 \mathrm{~Hz}$. The resulting 3D marker traces were then used for all analysis presented in this paper. The limb angle and length as well as the included angles for the sagittal plane limb, hip, knee and ankle joints were calculated. Due to the slight variations in time or number of data points per step, each step was divided into 25 bins ( $4 \%$ of the step cycle per bin), and the collected data points for each variable were averaged together in each bin. This resulted in a time normalization of each step, with each step having the same number of data points, and therefore, multiple steps could be averaged together. Nine normalized steps per trial were averaged together across 3 trials in the same condition and the means of each of the conditions were compared. In order to evaluate the yield during stance, we calculated the change in limb length and ankle angle during weight acceptance. 


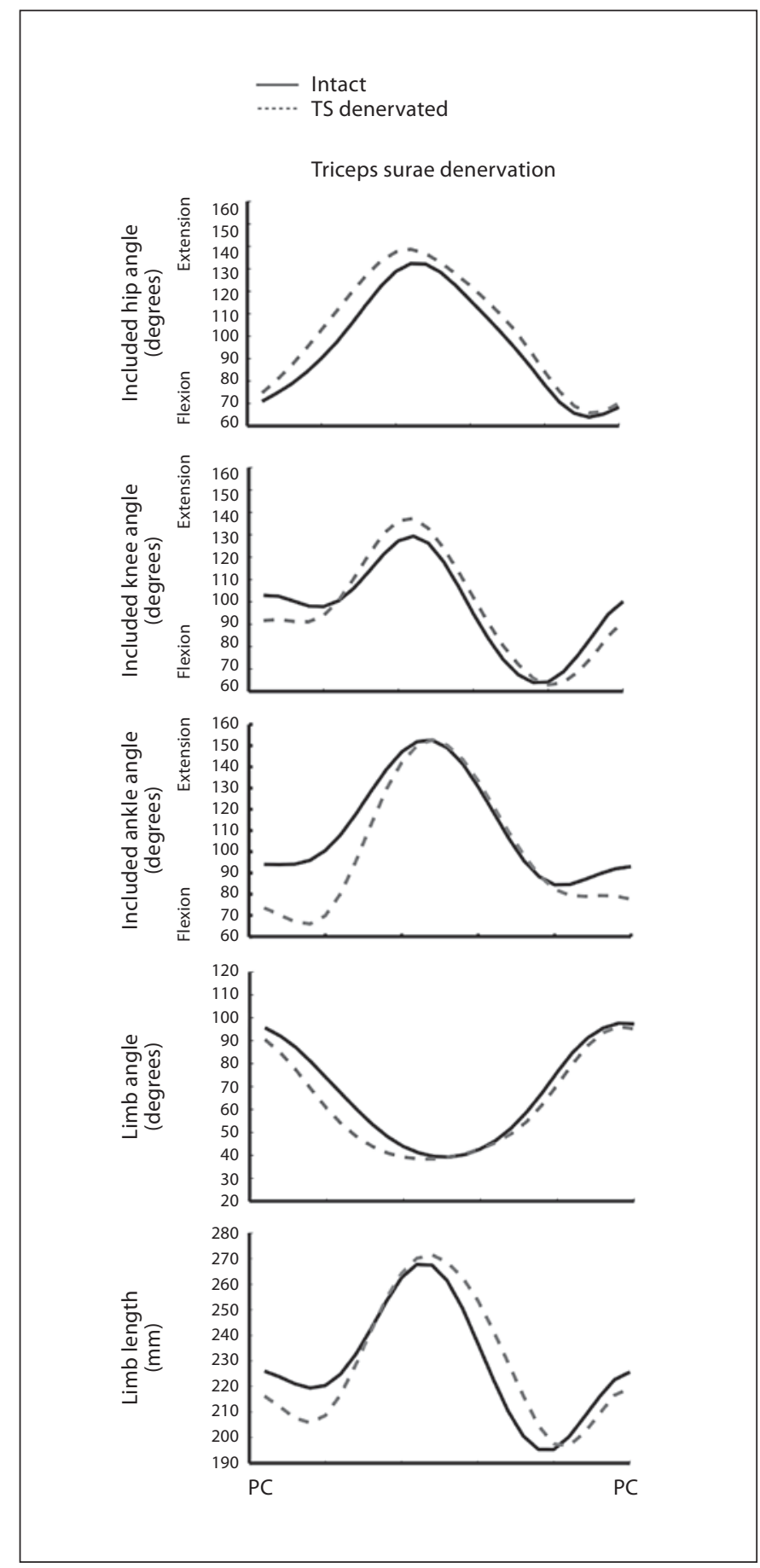

Fig. 2. The mean joint and limb trajectories in the sagittal plane are plotted for 1 cat over a time-normalized step cycle, from paw contact (PC) to paw contact. Black line $=$ Trajectories $(\mathrm{TS})$ from the intact limb; grey dashed line $=$ trajectories from the denervated limb. Note the greater ankle flexion at the beginning of stance and the increased yield. Note further the decrease in limb length during weight acceptance during stance.
The mean and standard deviations of each kinematic variable for each cat before and after triceps denervation or complete fasciotomy were plotted against each other. The line of identity was drawn through the graph. Points on this line would indicate that the treatment had no effect on the kinematic variable, while location of the mean values off the line of identity would indicate increases or decreases resulting from the treatment. In order to determine if there were significant changes across animals, a paired two-sample for means $t$ test was performed on the mean values of the kinematic variables $(\alpha=0.05)$.

The angular accelerations for the included joint angles over the step cycle were determined by differentiating the included angles twice prior to normalizing the data. The maximal angular acceleration of the ankle was averaged across all steps. The means of the angular accelerations before versus after treatment (triceps surae denervation or complete fasciotomy) were plotted against each other to show the trend in change of acceleration across all cats (as described for the angles).

The variance in the sagittal plane of the included hip, knee and ankle angles and limb length were evaluated as well as the frontal plane limb orientation. The summed variance was averaged under each condition, and the values before and after treatment (triceps denervation or complete fasciotomy) were plotted against each other.

\section{Electromyography}

While the animal was still under anesthesia, bipolar EMG wires were inserted into a number of muscles, including the plantaris (Plant) and flexor hallucis longus (FHL) muscles, in order to evaluate any neural adaptations that might have occurred over the time course of the experiment. The EMG recordings were made from lengths of insulated, stainless steel wire twisted together, with the last $3 \mathrm{~mm}$ of insulation stripped from the end and tied together using surgical silk with the ends offset from each other. These electrodes were then inserted and sutured into the muscle bellies. The recordings were collected at $1,000 \mathrm{~Hz}$ and preamplified by a gain of 100 .

The EMG recordings for FHL and Plant were rectified and filtered using a 4th-order, zero-lag Butterworth filter. The resulting signal was time normalized for each step and averaged across each condition using the same criteria as for the kinematics ( 9 steps for 3 trials per condition). The normalized mean EMG patterns were plotted and compared to see if there was an increase in duration or magnitude of activity of the muscles. A burst was defined as a signal $>2$ standard deviations above the background level.

\section{Results}

\section{Sagittal Plane Kinematics}

Upon the denervation of the triceps surae, the ankle was initially more flexed. In addition, there was an increase in ankle yield and a decrease in limb length during the weight acceptance stage of stance during level walking (fig. 2) in the sagittal plane. The weight acceptance stage of stance is the initial period of stance where the center of mass prepares to pass directly over the paw [Fowler et al., 1993]. In the example shown in figure 2, the 


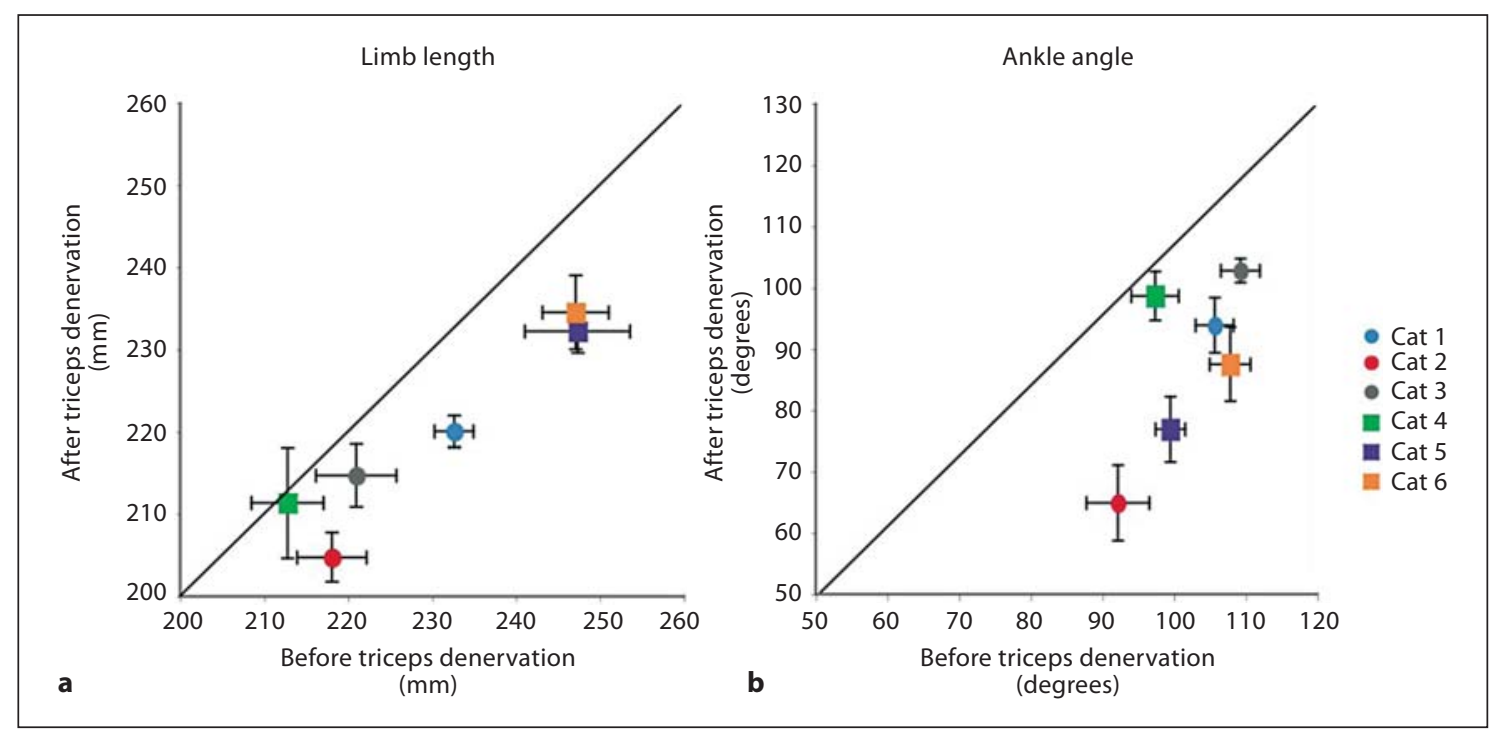

Fig. 3. The mean minimum limb length (a) and ankle angle (b) during weight acceptance plotted before versus after the denervation of the triceps surae. Note that in all but 1 case limb length decreased and in all cases ankle flexion decreased after denervation. Solid line = Line of identity; circles = experiments in which denervation was performed first; squares = experiments in which fasciotomy was performed first.

ankle was initially more flexed by about $20^{\circ}$, increased further into dorsiflexion by another $10^{\circ}$, leading to an increased dorsiflexion of $30^{\circ}$. Furthermore, the limb length decreased by $20 \mathrm{~mm}$. Although these large changes were observed in these two variables, the included hip and knee angles and the limb orientation angle remained conserved. An increase in knee extension was noted in some cases (fig. 2), but this increase was small and not significant.

The increase in dorsiflexion was observed across all cats and the decrease in limb length was observed for all but 1 of the cats following denervation of the triceps surae muscles (fig. 3). The increase in ankle dorsiflexion during weight acceptance was significant $(p=0.02)$. The decrease in limb length was also found to be significant $(p=0.005)$ independent of the order of treatments. Mean limb length and ankle angle were minimally affected by the crural fasciotomy (fig. 4). The limb length in particular was conserved. There was a trend toward an increase in ankle yield in 3 of the animals (fig. 4), but this increase was not significant $(p=0.37)$. In general, the effects of the two manipulations were independent of the order in which they were performed.

\section{Ankle Angle Acceleration}

The mean trajectories of the ankle angular acceleration for 2 cats are shown in figure 5a, b. Either the triceps surae muscles were denervated first or the crural fasciotomy was performed first. These traces line up with the timing of the included ankle angle in figure 2. The general pattern of acceleration was similar for all conditions. During the period of weight acceptance, the ankle accelerated for each condition (fig. 2, 5a, b, grey box) and then decelerated toward a maximum value achieved at toe off (fig. 5a, b, clear box). During the weight acceptance period (grey box), there was a trend toward an increase in acceleration upon the denervation of the triceps surae for 5 of the cats, with or without a preceding fasciotomy (fig. 5c, upper left panel). This reason for the increase in acceleration is apparent from figure 2 (included ankle angle). The increased ankle yield delayed the extension, resulting in a more pronounced acceleration during the extension. During paw off (clear box), there was a corresponding increase in deceleration after the denervation of the triceps surae in 3 of the animals, resulting in preservation of the duty cycle (fig. 2, 5e).

While the denervation of the triceps surae resulted in increased acceleration during weight acceptance and deceleration at paw off, the complete fasciotomy resulted in the opposite pattern, a decrease in acceleration during weight acceptance (fig. 5d) and a decrease in deceleration at paw off. The decrease in acceleration during weight acceptance after the complete fasciotomy was observed in 5 cats. However, there was not a consistent 


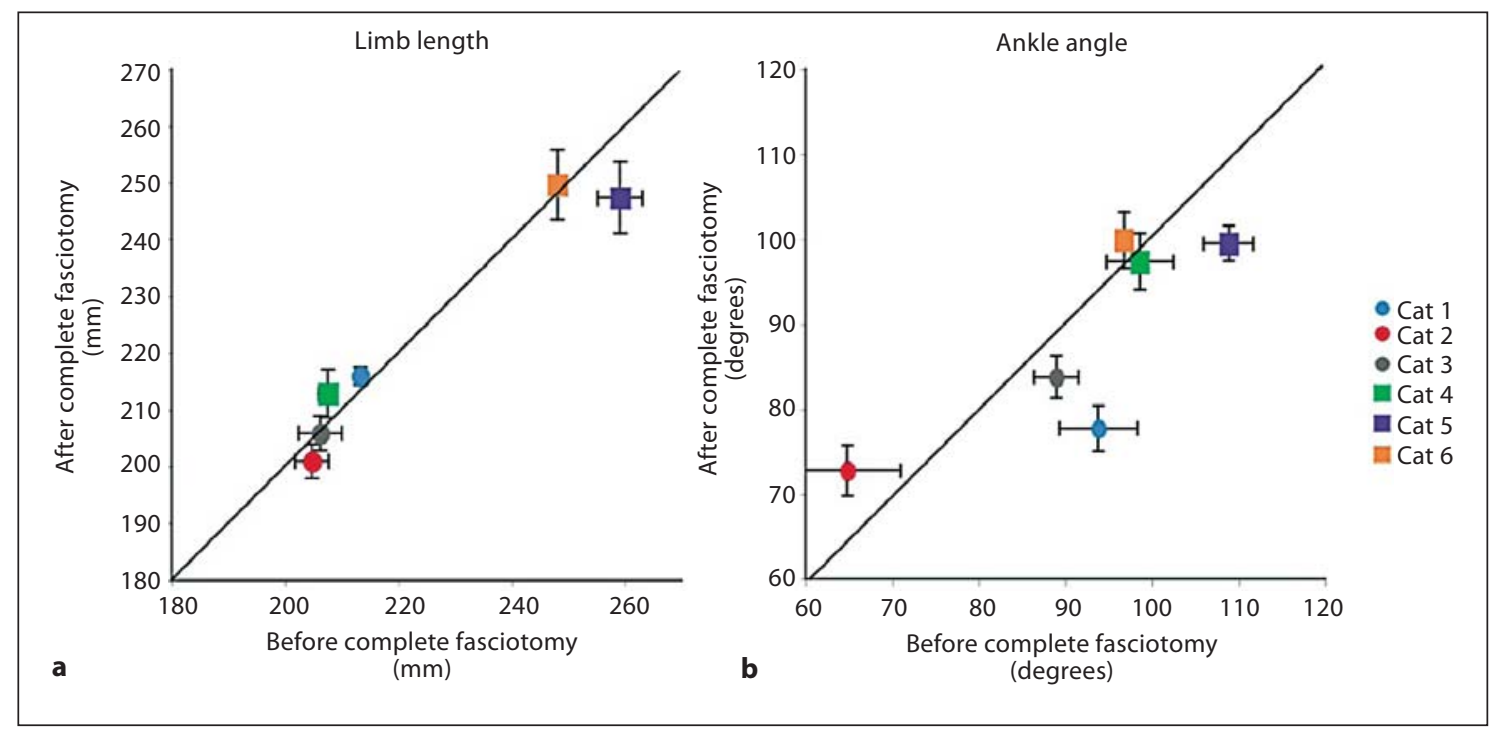

Fig. 4. The mean limb length (a) and ankle angle (b) during weight acceptance plotted before versus after complete fasciotomy. Limb length is preserved in most cases following fasciotomy (a). Increased flexion of the ankle was observed in 3 cases, and decreased flexion was observed in 1 case (b). Solid line = Line of identity; circles = denervation performed first; squares = fasciotomy performed first.

or significant change in the ankle yield. The decrease in deceleration at paw off was observed in 5 cats - also documented in Stahl [2010] - although there was no change in net ankle angular excursion. The effects of denervation and fasciotomy on angular acceleration were independent of the order in which these treatments were performed.

\section{Variance}

The denervation of the triceps surae resulted in an increase in summed variance for the joint and sagittal plane limb angles (fig. 6). Variances of the hip, knee and ankle angles increased for all cats, while the sagittal limb orientation angle showed only a modest increase. The limb length variance also increased for 4 of the animals. The frontal limb angle did not show a consistent trend in variance after the triceps surae denervation, with 3 cats increasing in variance and 3 cats decreasing in variance, suggesting that the frontal plane may rely more on passive mechanical elements such as fascia for variance control [Stahl, 2010].

\section{EMG Recordings}

The EMG activities for the recorded muscles did not show significant changes after denervation of the triceps surae muscles. The data for Plant and FHL are shown in figure 7 . These muscles contribute to ankle plantarflex- ion, and therefore, would be the most likely to show compensatory increases in activity following denervation of the triceps surae muscles. The mean EMG traces for 3 cats are plotted in figure 7 and show that after triceps surae denervation the mean activity remained within the range of the intact values. Although there was some variability in the amplitudes of muscular activity, the durations of the activity were little affected by the treatments. However, in view of the large standard deviation of the EMG signals, more subtle changes due to the treatment would not be detected.

\section{Discussion}

\section{Summary}

We tested the hypothesis that the trajectories of the task level variables, limb length and limb orientation, would be preserved following denervation of the triceps surae muscles by rapid sensory feedback or intrinsic mechanical properties of the limb. We further hypothesized that sensory feedback from retractor muscles (muscles that rotate the limb backwards and contribute to propulsion) of the thigh could provide the necessary feedback of limb length and orientation by virtue of the mechanical linkage of these muscles to the calcaneus by way of the crural fascia. We employed the spontaneously stepping 


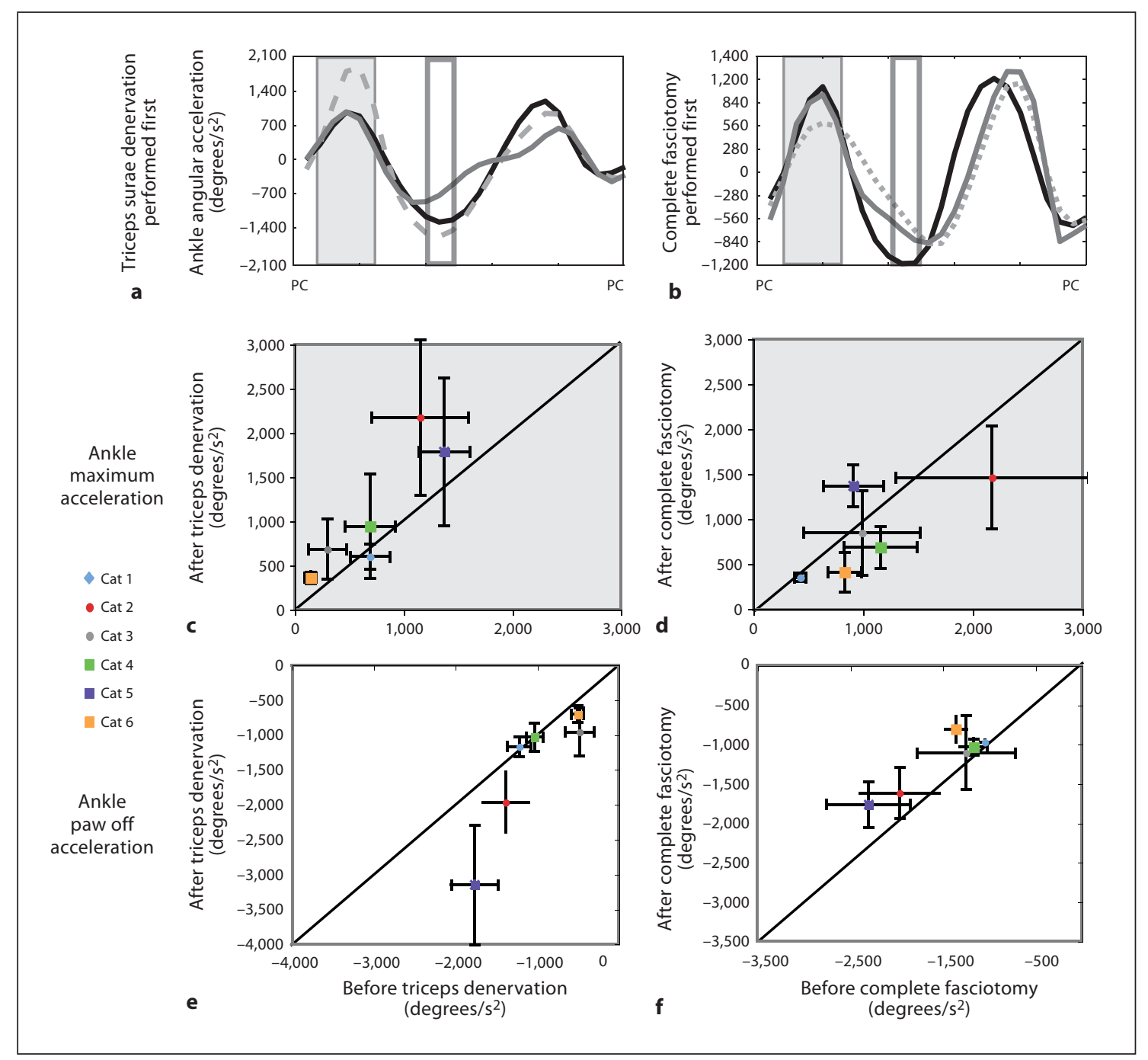

Fig. 5. a, b Mean angular acceleration of the ankle through the step cycle for 2 representative cats. PC = Paw contact. a Effects of treatments when triceps denervation was performed first. Black line = Intact; grey dashed line = triceps denervated; solid grey line $=$ complete fasciotomy. $\mathbf{b}$ Effects of treatments when fasciotomy was performed first. Black line = Intact; grey dashed line = fasciotomy; solid grey line $=$ triceps denervation; dark grey box $=$ time period of weight acceptance, corresponding to graphs in $\mathbf{c}$, d; clear box $=$ time period of paw off, corresponding to the analy-

decerebrate cat preparation because the immediate effects of the treatments could be assessed without surgical recovery and the possibility of longer-term adaptive changes in motor control. We found that denervation of the triceps surae muscles resulted in increases in ankle yield and total dorsiflexion as well as in a subsequent increase in acceleration as the ankle extended toward paw off. In addition to the increased ankle yield, we found that

Muscular Denervation and Fasciotomy Affect Global Limb Variables sis shown in e, f. c-f The mean and standard deviations of the angular accelerations before versus after treatment are applied. The denervation of the triceps surae resulted in an increase in acceleration during weight acceptance in 4 cases (c) and in an increase in deceleration at paw off in 4 cases (e). Complete fasciotomy resulted in a decrease in acceleration during weight acceptance in 3 cases (d) and in a decrease in deceleration at paw off in 4 cases (f). Circles $=$ Denervation performed first; squares $=$ fasciotomy performed first.

limb length was not preserved over the time course of these experiments and decreased significantly during the weight acceptance phase of the step cycle. Limb length and ankle yield were minimally affected by crural fasciotomy, regardless of the order in which the two disruptions were performed. Therefore, the results do not support either one of the above hypotheses. The fasciotomy did not result in a significant change in ankle yield or 


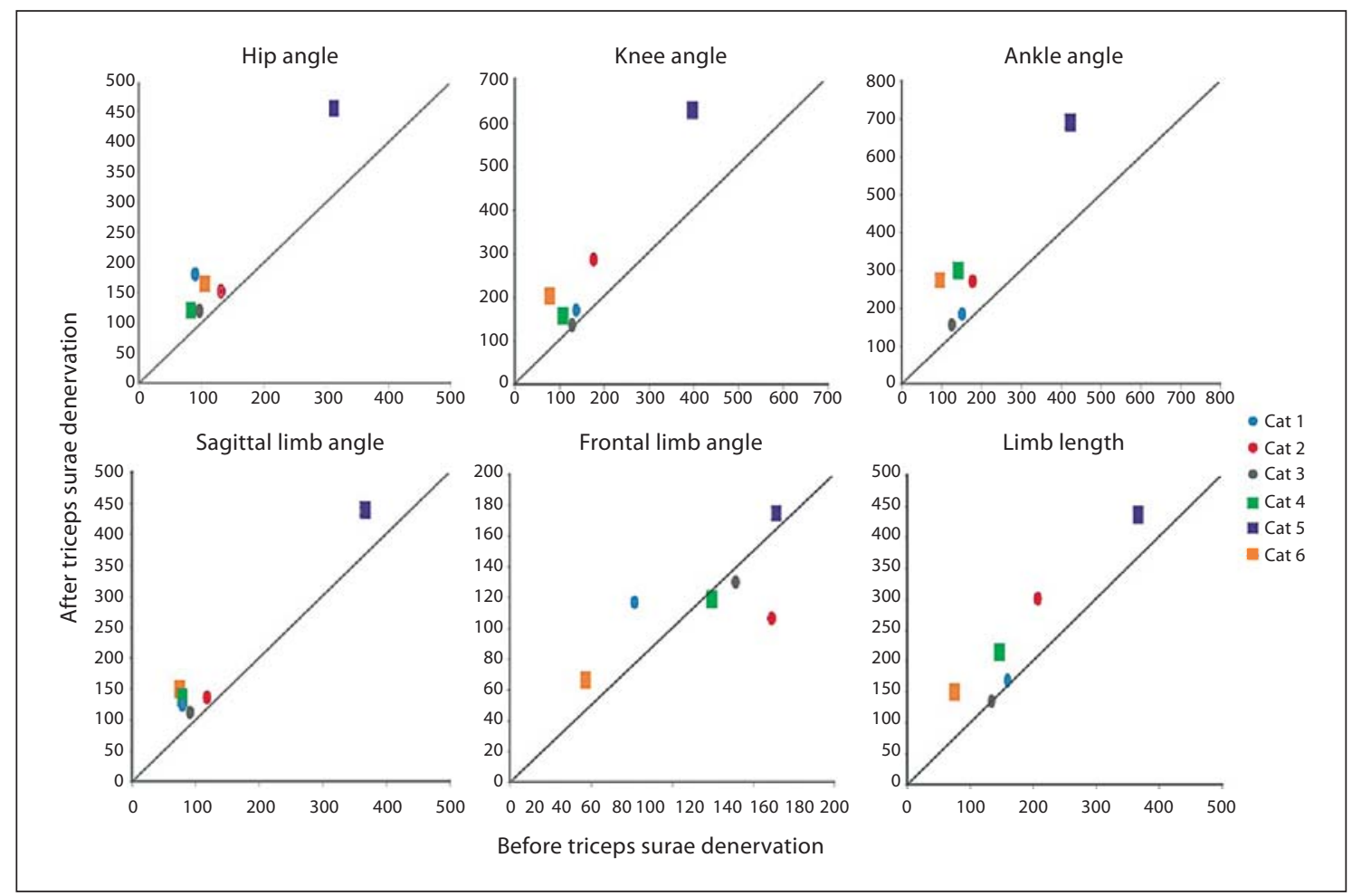

Fig. 6. Change in summed variance of joint and limb variables following denervation of the triceps surae muscles for each cat. The denervation of the triceps surae resulted in an increase in mean summed variance for the joint and limb variables in the sagittal plane for most experiments. A variable response was ob- served for the limb angle in the frontal plane with 3 cases showing increases and 3 cases showing decreases in variance. Hip, knee, ankle, sagittal limb and frontal limb angles are given in degrees; limb length is given in $\mathrm{mm}$. Circles = Denervation performed first; squares $=$ fasciotomy performed first. limb length during weight acceptance but did show a trend toward a decrease in maximum acceleration during weight acceptance and at paw off. These results confirm that the triceps surae are strong antigravity muscles during weight acceptance while the hamstring muscles through the crural fascia assist with propulsion throughout stance.

\section{Comparison to Non-Decerebrate Cat Kinematics}

A comparison of the data reported here with the results of the chronic studies reviewed in the introduction provides an important clue concerning the adaptation to muscular denervation. Both acute and chronic preparations in which the triceps surae nerves were transected show similar results during the paralytic stage of the triceps surae at the joint variable level but differ at the limb level with the lack of conservation of limb length being only observed in the decerebrate preparation. The included sagittal plane angles following denervation of the triceps surae muscles show similar effects and ranges of an- gles between the non-decerebrate [Chang et al., 2009] and decerebrate cat (presented in this paper). The results presented here also showed a similar increase in ankle yield to that reported 1-2 weeks after denervation of the MG synergists [Pearson et al., 1999] or soon after denervation with nerve repair of MG and LG [Maas et al., 2007].

In contrast, the joint and limb variables were not conserved after the denervation of the triceps surae in our study. In particular, we observed increased ankle dorsiflexion and a decrease in limb length during weight acceptance. In the awake, behaving cat, ankle dorsiflexion increased while limb length and orientation were conserved 1 month after denervation of the triceps surae muscles, before significant reinnervation was able to take place [Chang et al., 2009]. The decrease in limb length in the acute preparation suggests that regulation of the limb variables requires a process of neural adaptation as is the case for the recovery of ankle yield in the chronic animal [Pearson et al., 1999] and is not regulated by ongoing feedback in the spinal cord. Intrinsic compensatory 
Fig. 7. The mean time-normalized EMG traces for FHL and Plant are plotted from paw contact (PC) to paw contact. Black line $=$ Mean values of EMG for the intact limb; black dotted lines = standard deviation for that mean; dark grey line $=$ mean EMG trace after denervation of the triceps surae; light grey line = mean EMG after complete fasciotomy (cat No. 4 only). Overall, no consistent change in EMG pattern was detected as a result of the denervations.

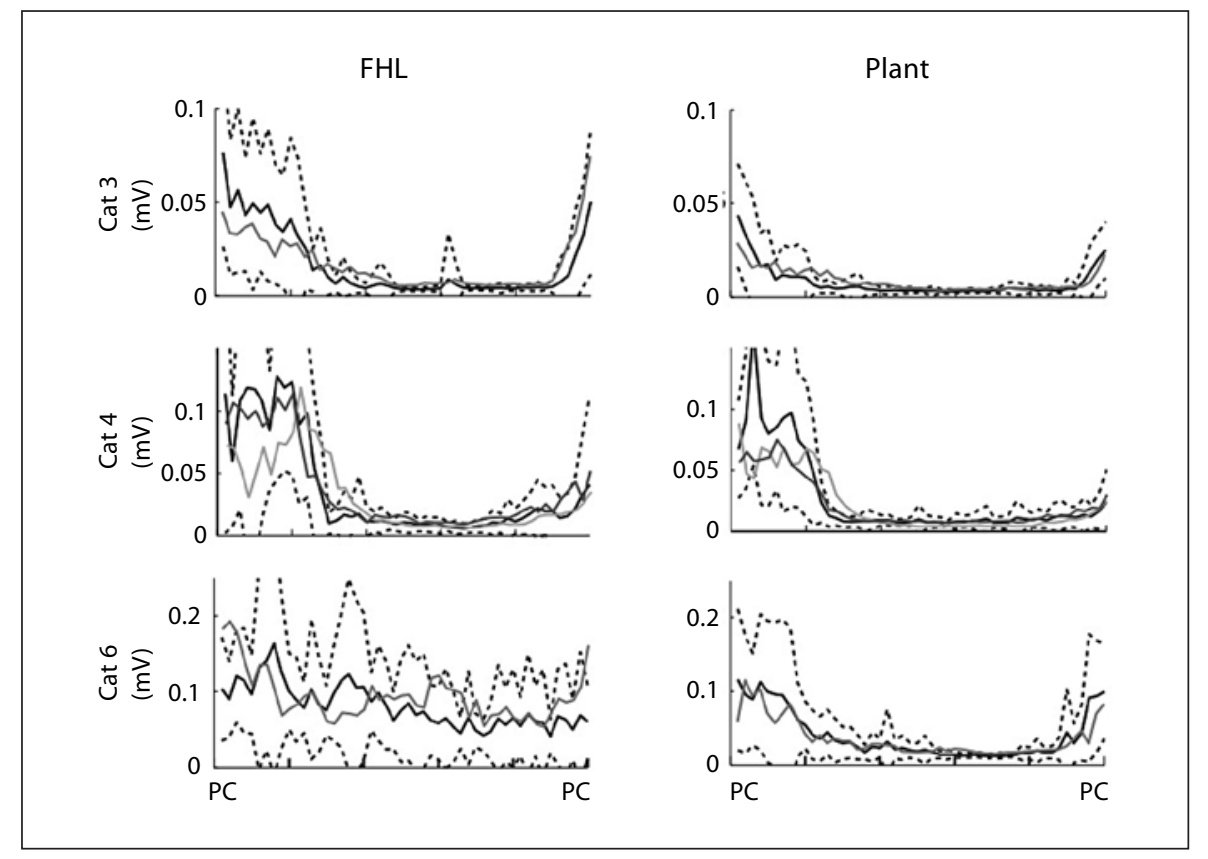

mechanisms were also ruled out. The slight increase in knee extension following paralysis of the triceps surae muscles was not significant and in any case too small to provide adequate compensation.

The greater increase in variance observed for the joint variables over the limb variables following denervation of the triceps surae muscles supports the idea that the limb variables are more conserved than the joint variables following adaptation [Chang et al., 2009]. In the cases of acute denervation reported here, increases in variance of the task level variables also occurred, corresponding to the lack of conservation of these variables in the short term. This difference further supports the hypothesis that compensation for the increases in ankle flexion and limb length requires longer-term adaptation. Interestingly, the frontal limb angle had an inconsistent trend with both increases and decreases in variance suggesting that there may be additional influences on the mediolateral stability of the limb.

\section{Role of the Triceps Surae and Crural Fascia during Step Cycle}

Weight Support Stage of Stance

During weight support, the increase in ankle dorsiflexion angle and acceleration after denervation of the triceps surae muscles suggests a loss of the antigravity action of these muscles within the limb in the cat. This supports results from previous work in humans that showed that during mid-stance, the triceps surae muscles provide vertical limb support during walking [Neptune et al., 2001]. Although the gastrocnemius muscles are biarticular, there was only a slight increase in knee extension (data not shown) suggesting that during weight acceptance the gastrocnemius muscles act more to extend the ankle and less to flex the knee [Lan and Crago, 1992]. However, an increase in knee extension corresponding to the increased ankle dorsiflexion was observed for downramp walking in the chronic cat 9-12 months after reinnervation of the triceps surae [Abelew et al., 2000]. This increased knee extension was presumed to compensate for the increased ankle yield observed in downhill walking and further suggests that the task level variables of limb length and orientation are regulated by an adaptive, neural process.

The complete disruption of the crural fascia did not significantly affect the ankle yield or the limb length suggesting that it does not play an antigravity role in the limb during weight acceptance. However, a decrease in acceleration was observed. The decrease in acceleration after complete fasciotomy can be attributed to a loss of limb retraction (backward motion of the limb during propulsion). The loss of the connection of the limb retractors or hamstring muscles to the ankle joint resulted in a loss of retraction force applied to the calcaneus and an increase in force applied more proximally on the tibia. This resulted in the potential for greater knee flexion and less 
extension of the ankle through weight acceptance, and thus, a loss in propulsion. Therefore, although the hamstring and crural fascia complex has a similar termination point to the triceps surae, the triceps surae muscles play a greater role in weight support during the weight acceptance phase than the hamstring muscles with crural fascia.

\section{Propulsive Stage of Stance}

During the propulsive stage of stance, deceleration increased after denervation of the triceps surae muscles, which is associated with the increase in angular excursion of the ankle joint through stance. Previous studies in human subjects [Neptune et al., 2001; Gottschall and Kram, 2003] have shown that throughout the stance phase, the soleus muscle provides weight support while the gastrocnemius muscles are initially weight supporting, but by mid-stance, become primarily propulsive. The increase in deceleration at paw off can be attributed to a 'catch-up' mechanism that maintains normal duty (fraction of the step cycle occupied by stance) in response to the increased ankle yield, rather than to a contribution to propulsion. This phenomenon might be associated with treadmill locomotion, where the cadence is influenced by treadmill locomotion. During overground locomotion, propulsion might be influenced.

\section{Implications for Neuronal Mechanisms of Adaptation}

Joint kinematics are sensed through limb proprioception, either by stretch of muscle receptors and possibly by joint receptors [Boyd and Roberts, 1953]. The presumed greater stretch and force in the FHL and Plant following denervation of the triceps surae would be expected to have led to an increase in EMG of these synergists independently of any adaptive increases. Our inability to detect a significant increase in the activation of these muscles was perhaps due to the variability of these signals across steps. However, the results do indicate that any substantial adaptation did not occur over the time course of these experiments. Furthermore, it has been shown that after the denervation of LG and soleus muscles during level walking there is little or no stretch of the MG fascicles. The stretch of the muscle tendon unit is accounted for mainly by stretch of the in-series tendon [Maas et al., 2010]. In a similar manner, we may not have observed an increase in stretch of Plant and FHL due to the long tendons and short fascicles of these muscles.

Recordings from neurons in the dorsal spinocerebellar tract have shown to relay limb length and orientation information to the cerebellum [Bosco et al., 2000], a structure known to contribute to adaptations of reflex pathways such as the vestibulo-ocular reflex. Although the cerebellum is conserved in the premammillary decerebration, the decerebration procedure disrupts a massive cortical input. Furthermore, any compensation through spinocerebellar pathways may require days or weeks in order for the cerebellum to respond to the change in the variable. It is known that the vestibulo-ocular reflex takes at least 5 days to change the gain in response to the unilateral removal of the vestibular nerve [Mailoli and Precht, 1985]. Our results characterized the immediate response of the limb to the injury, and therefore, by design, did not allow for a period of adaptation that may have taken days or weeks. Generally, the data were collected within approximately $30 \mathrm{~min}$ of the denervation of the triceps surae while the paralytic stage in non-decerebrate animals was 4 weeks after injury [Chang et al., 2009]. It was recently shown in experiments utilizing partial denervation of the triceps surae and Plant that limb length was preserved 1 day following surgery, suggesting that mechanisms preserving global variables can effect substantial compensation within $24 \mathrm{~h}$ [Hart et al., 2010].

\section{Control of Stance-to-Swing Transition}

At least two sensory systems are thought to contribute to the termination of stance and the initiation of swing: signals from Golgi tendon organs in the gastrocnemius muscles signaling unloading of the ankle [Pearson, 2008] and signals from muscle spindles in the hip flexors [Grillner and Rossignol, 1978]. Given that the trajectories of the included angle of the hip and of the limb orientation angle were conserved under the different interventions to the distal limb, we can conclude that there are redundant sensory systems within the limb that allow for the conservation of these variables. However, the hip flexors are mostly unaffected by both denervation and disruption of the crural fascia, and thus, still provide sensory information to initiate the swing phase. Although we did note a slight increase in hip extension after triceps denervation, it was not sufficient to alter the timing of the stance to swing transition.

\section{Acknowledgments}

The authors thank Christopher Tuthill, Claire Honeycutt, $\mathrm{PhD}$, and Inez Falcon for their assistance during experiments, William Goolsby for his technical support and Michael Kutner, $\mathrm{PhD}$, for his statistical support. Financial support was provided by National Institutes of Health/National Institute of Child Health and Human Development grant HD32571. 


\section{References}

Abelew, T.A., M.D. Miller, et al. (2000) Local loss of proprioception results in disruption of interjoint coordination during locomotion in the cat. J Neurophysiol 84: 2709-2714.

Bosco, G., R.E. Poppele, et al. (2000) Reference frames for spinal proprioception: limb endpoint based or joint-level based? J Neurophysiol 83: 2931-2945.

Boyd, I.A., T.D. Roberts (1953) Proprioceptive discharges from stretch-receptors in the knee-joint of the cat. J Physiol 122: 38-58.

Chang, Y.H., A.G. Auyang, et al. (2009) Whole limb kinematics are preferentially conserved over individual joint kinematics after peripheral nerve injury. J Exp Biol 212: 35113521.

Fowler, E.G., R.J.G. Gregor, J.A. Hodgson, R.R. Roy (1993) Relationship between ankle muscle and joint kinetics during the stance phase of locomotion in the cat. J Biomech 26: $465-$ 483.

Gottschall, J.S., R. Kram (2003) Energy cost and muscular activity required for propulsion during walking. J Appl Physiol 94: 17661772 .
Grillner, S., S. Rossignol (1978) On the initiation of the swing phase of locomotion in chronic spinal cats. Brain Res 146: 269-277.

Hart, K.S., P. Nardelli, T.C. Cope (2010) Neural compensation for partial synergist denervation (abstract). Soc Neurosci Abstr 36:381.23.

Lan, N., P.E. Crago (1992) A noninvasive technique for in vivo measurement of joint torques of biarticular muscles. J Biomech 25: 1075-1079.

Maas, H., R.J. Gregor, et al. (2010) Locomotor changes in length and EMG activity of feline medial gastrocnemius muscle following paralysis of two synergists. Exp Brain Res 203 681-692.

Maas, H., B.I. Prilutsky, et al. (2007) The effects of self-reinnervation of cat medial and lateral gastrocnemius muscles on hindlimb kinematics in slope walking. Exp Brain Res 181: 377-393.

Mailoli, C., W. Precht (1985) On the role of vestibulo-ocular reflex plasticity in recovery after unilateral peripheral vestibular lesions. Exp Brain Res 59: 267-272.

Neptune, R.R., S.A. Kautz, et al. (2001) Contributions of the individual ankle plantar flexors to support, forward progression and swing initiation during walking. J Biomech 34: 1387-1398.
Pearson, K.G. (2008) Role of sensory feedback in the control of stance duration in walking cats. Brain Res Rev 57: 222-227.

Pearson, K.G., K. Fouad, et al. (1999) Adaptive changes in motor activity associated with functional recovery following muscle denervation in walking cats. J Neurophysiol 82 : $370-381$.

Stahl, V.A. (2010) A Biomechanical Analysis of the Crural Fascia in the Cat Hindlimb; doctoral dissertation, Georgia Institute of Technology, Atlanta.

Stahl, V.A., C.F. Honeycutt, T.R. Nichols (2008) Dissecting force inputs from calcaneal structures: determining the propulsive and stabilizing roles of the crural fascia and triceps surae (abstract). Washington, Society for Neuroscience 34:377.7.

van Ingen Schenau, G.J. (1994) Proposed action of bi-articular muscles and the design of hindlimbs of bi- and quadrupeds. Hum Mov Sci 13: 665-681. 